\title{
Variable stars in the VVV globular clusters
}

\author{
Javier Alonso-García ${ }^{1,2, \star}$, Márcio Catelan ${ }^{3,2}$, Rodrigo Contreras Ramos ${ }^{2,3}$, István Dékány ${ }^{4}$, and \\ Dante Minniti ${ }^{5,2,6}$ \\ ${ }^{1}$ Universidad de Antofagasta, Unidad de Astronomía, Avda. U. de Antofagasta 02800, Antofagasta, Chile \\ ${ }^{2}$ Millennium Institute of Astrophysics, Santiago, Chile \\ ${ }^{3}$ Pontificia Universidad Católica de Chile, Instituto de Astrofísica, Av. Vicuña Mackenna 4860, 782-0436 \\ Macul, Santiago, Chile \\ ${ }^{4}$ Zentrum für Astronomie der Universität Heidelberg, Astronomisches Rechen-Institut, Mönchhofstr. 12-14, \\ D-69120 Heidelberg, Germany \\ ${ }^{5}$ Universidad Andrés Bello, Departamento de Física, Av. Fernández Concha 700, Las Condes, Santiago, \\ Chile \\ ${ }^{6}$ Vatican Observatory, V00120 Vatican City State, Italy
}

\begin{abstract}
The VVV survey observed some of the most crowded and most obscured regions in the inner Milky Way during the last years. A significant sample of the less known globular clusters in our galaxy lie there. Combining the high-resolution, wide-field, nearinfrared capabilities of the survey camera, the use of 5 different filters, and multi-epoch observations, we are able to overcome many of the previous challenges that prevented a proper study of these objects. Particularly, the identification of the RR Lyrae stars in these globular clusters is proving to be a fundamental tool to establish accurately their distances and reddenings, and to infer information about the Oosterhoff dichotomy that Galactic globular clusters seem to follow.
\end{abstract}

\section{Introduction}

The globular clusters that lie in the inner regions of the Milky Way present important difficulties to extract accurate information about their physical parameters and about their stellar populations. The high extinction perceivable when observing towards the inner Galaxy, the high number of field stars in the region which increases the considerable crowding when observing these compact objects and complicates the proper identification of cluster stars, or the presence of some of the most poorlypopulated globular clusters in our Galaxy, are among the most significant of them.

The Vista Variables in the Vía Láctea (VVV) survey has been observing the inner regions of the Milky Way during the last 6 years. VVV is one of the ESO Public Surveys conducted with the $4 \mathrm{~m}$ VISTA telescope at the Paranal Observatory, in Chile. It surveyed a region of 562 square degrees in the region of the Galactic bulge and an adjacent region of the southern Galactic disc ([4]). There are 36 known Galactic globular clusters in the surveyed area, plus a few more new candidates $([3,5])$. The VVV survey allows us not only to observe these globular clusters from their very centers out to their tidal radii and beyond at near-infrared wavelengths were the effects of extinction are highly diminished, but also to search for any variable sources present thanks to its multi-epoch observations.

^jalonso@uantof.cl 

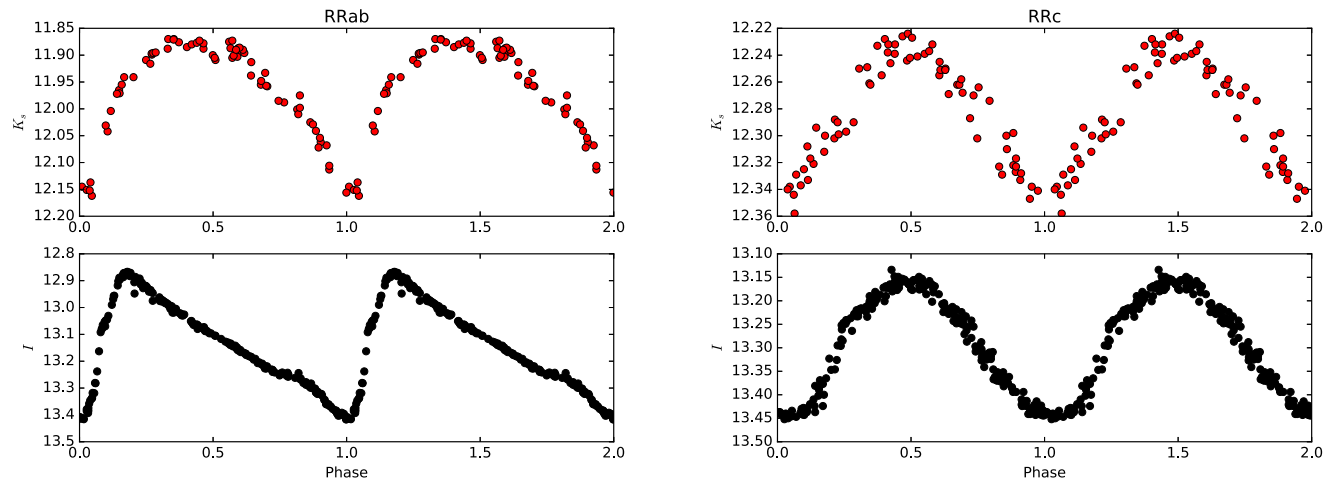

Figure 1. Light-curves of two RR Lyrae stars in NGC 6656 (M 22), one of the globular clusters in our sample. On the left, light-curve of V4, an RRab star; on the right, light-curve of V19, an RRc star. In the upper and lower panels, the $K_{s^{-}}$and $I$-band light-curves are shown, built with VVV and OGLE data ([6]), respectively. Note the change in the amplitudes and shapes between the different wavelengths considered.

\section{RR Lyrae in the VVV globular clusters}

RR Lyrae constitute a common type of variable stars in Galactic globular clusters. The tight periodluminosity relations they show, especially at near-infrared wavelengths, confer them an additional interest, as they can be used as standard candles to infer information about the physical parameters of the globular clusters they belong to. Their proper identification, however, is complicated by the fact that they show smaller amplitudes and more sinusoidal shapes in their near-infrared light-curves, when compared to the optical ones (see Fig. 1).

We were able to overcome these difficulties and identify a significant number of these variables in two of the most reddened globular clusters in our sample: 2MASS-GC02 and Terzan 10 ([1]). We found both clusters to be further away from us, and much closer to the Galactic center than previously reported. We also found the extinction towards them to be non-canonical, and their Oosterhoff classification uncommon for Galactic globular clusters ([2]). We are currently exploring the cases of several more inner Galactic globular clusters to check the accuracy of their literature physical parameters, and how they fit in the Oosterhoff dichotomy paradigm that seems to be present in our Galaxy.

Acknowledgments: Support is provided by the Ministry for the Economy, Development and Tourism, Programa Iniciativa Científica Milenio grant IC120009, awarded to the Millennium Institute of Astrophysics (MAS). J.A-G. acknowledges support from FONDECYT Iniciación grant 11150916.

\section{References}

[1] Alonso-García, J., Dékány, I., Catelan, M., et al., AJ, 149, 99 (2015)

[2] Catelan, M., Ap\&SS, 320, 261 (2009)

[3] Minniti, D., Hempel, M., Toledo, I., et al., A\&A, 527, A81 (2011)

[4] Minniti, D., Lucas, P. W., Emerson, J. P., et al., New Astronomy, 15, 433 (2010)

[5] Minniti, D., Palma, T., Dékány, I., et al, ApJL, 838, L14 (2017)

[6] Soszyński, I., Udalski, A., Szymański, M. K., et al., Acta Astronomica, 64, 177 (2014) 